\title{
Inversão da equação de Kozeny-Carman para meios porosos fractais
}

Átila Saraiva Quintela Soares*(IGEO-UFBA) \& Joelson Conceição Batista(IGEO-UFBA)

Copyright 2018, SBGf - Sociedade Brasileira de Geofísica Este texto foi preparado para a apresentação no VIII Simpósio Brasileiro de Geofísica, Salinópolis, 18 a 20 de setembro de 2018. Seu conteúdo foi revisado pelo Comitê Técnico do VIII SimBGf, mas não necessariamente representa a opinião da SBGf ou de seus associados. É proibida a reprodução total ou parcial deste material para propósitos comerciais sem prévia autorização da SBGf.

\section{Resumo}

Numerical modeling of petrophysical data is of great importance nowadays, when exploration and studies of nonconventional reservoir require models that better capture its complexity. In this context, using a generalized equation of Kozeny-Carman for fractal porous medium, an inversion of permeability data, from core analysis taken from offshore wells located in the Norwegian sect on the North Sea. The used inversion method was Differential Evolution, which was modified in order to with the intent of increasing the search radius for global minimum. After using the algorithm, it was possible to adjust the experimental points with satisfactory error, reaffirming its validity. In this manner, it was possible to calculate parameters that allow permeability to be described as a function of porosity, being a good alternative to well log and petrophysical data correlation.

\section{Introdução}

Estudos petrofísicos recentes, incluindo modelagem de meios porosos complexos tais como os reservatórios não convencionais, denominados de Shale Gas/Oil, tem se tornado alvo de muitos estudos (e.g. YU et al., 2014; BUST et al., 2013). Esses reservatórios são formados por folhelhos e que devido aos baixos valores de porosidade, permeabilidade e sua irregular distribuição das acumulações de hidrocarbonetos ao longo do reservatório, não produz naturalmente a taxas econômicas, tendo sua exploração viável caso seja realizada a combinação de perfuração de poços horizontais/multi-laterais com o processo de estimulação por fraturamento hidráulico. O conhecimento petrofísico deste tipo de litologia, através de amostragem de rochas em poços perfurados, pode contribuir para a reutilização destes resultados na interpretação dos poços em que foram extraídas tais amostras.

A correlação de parâmetros petrofísicos laboratórial e de poços é essencial na caracterização dos reservatórios não convencionais. Bust et al. (2013) aplicou essa correlação nos reservatórios de Shale gas, visto que é formado por uma litologia com baixa permeabilidade e alta argilosidade, o que dificulta o cálculo utilizando medidas de perfilagem, necessitando de correlação com dados experimentais de laboratório. Da mesma forma, reservatórios carbonáticos podem ser auxiliados por dados experimentais por apresentarem dificuldades similares.

Esse trabalho tem como objetivo utilizar a equação de Kozeny-Carman (KC) generalizada elaborada por Brêttas (2010), que não apresentam as limitações dessa equação clássica e se basea na Teoria dos Meios Fractais, fornecendo a relação entre porosidade e permeabilidade. O modelo que generaliza a equação de Kozeny-Carman, fornece uma relação existente entre porosidade e permeabilidade de diversos tipos de materiais porosos, contudo é necessário na prática estimar alguns parâmetros que varia conforme a amostra utilizada. Assim, a partir de alguns valores experimentais medidos de porosidade e permeabilidade para uma determinada amostra, podemos estimar o valor dos parâmetros para cada amostra em questão. Dessa maneira, é possível obter uma função do tipo $K(\phi)$ que corresponde a equação de Kozeny-Carman generalizada para uma dada amostra, sendo que a estimativa dos parâmetros do modelo foi realizada utilizando uma estratégia de otimização via um algorítimo de Evolução Diferencial modificado. Nesse algorítimo minimiza-se a diferença entre os valores calculados de permeabilidade, a partir da equação de KC, e os valores experimentais. Esse procedimento torna possível estimar os parâmetros da equação KC.

\section{Teoria e Metodologia}

Nesta seção abordaremos a equação de Kozeny-Carman generalizada e o método de otimização implementado. Os dados laboratoriais utilizados também serão descritos nessa seção.

\subsection{A equação de Kozeny-Carman generalizada}

A equação de Kozeny (1927) surgiu como uma proposta de estudo para um problema que perdura até hoje, o de como avaliar a permeabilidade a partir das características geométricas dos poros. Seelheim (1880) afirmou que a permeabilidade deveria ser expressa pelo quadrado de algum diâmetro característico do meio poroso. Desde então vários trabalhos utilizando métodos estatísticos e empíricos propuseram vários modelos, e aparentemente os mais eficientes utilizavam três parâmetros, como por exemplo tamanho dos poros, tortuosidade e conectividade (CHAPUIS; AUBERTIN, 2003).

Mais tarde modificada por Carman $(1937,1938)$, a equa- 
ção de Kozeny (1927), passou a ser chamada de equação de Kozeny-Carman (KC) e surgiu como uma solução que considerava o meio poroso como um conjunto de tubos capilares. Nesses tubos foi utilizada a equação de NavierStrokes, levando em conta a sua tortuosidade. Essa equação teve várias formas, incluindo a seguinte, e que é muito utilizada:

$$
\sqrt{\frac{k}{\phi}}=\frac{r}{6 \sqrt{2 \tau}}\left(\frac{\phi}{1-\phi}\right),
$$

onde,

$$
c=\frac{r}{6 \sqrt{2 \tau}}>0
$$

é o coeficiente de Kozeny, $\tau$ é a tortuosidade, $r$ é o raio dos grãos e $\phi$ a porosidade. Observou-se que essa equação não é utilizável para meios porosos impermeáveis, pois o coeficiente $c$ nunca se anula. A dedução dessa equação pode ser encontrada no trabalho de Brêttas (2010).

Porém, desde então surgirão várias tentativas de generalização dessa equação para meios porosos mais complexos e compactados (e.g. COSTA, 2006; BRÊTTAS, 2010). No trabalho de Brêttas (2010), a equação de KC foi generalizada utilizando a teoria fractal. Partindo de algumas hipóteses, Brêttas (2010) considera que a superfície específica e a tortuosidade podem ser expressas pela lei fractal, conforme as equações a seguir:

$$
\begin{gathered}
\frac{1}{M_{b}}=C_{1 / M_{b}}(1-\phi)^{-D_{1 / M_{b}}} \\
\tau=C_{r} \phi^{-D_{r}}
\end{gathered}
$$

onde, conforme a equação $3, C_{1 / M_{b}} \geqslant 0$ e $D_{1 / M_{b}} \geqslant 0$ são respectivamente o coeficiente e dimensão fractal de $1 / M_{b}$, e $M_{b}$ é a superfície específica. Na equação 4, de forma análoga, $C_{r}$ e $D_{r}$ são, respectivamente, o coeficiente e dimensão fractal do raio poroso $(r)$. Além dessas hipóteses, o meio poroso é considerado como um pacote de vários tubos capilares, porém não necessariamente de seção transversal circular, assim com o escoamento é dado pela lei de Hagen-Poiseuille. Além disso, se considera que o raio hidráulico dessa lei é igual a razão entre a porosidade e a superfície específica. Utilizando essas hipóteses, Brêttas (2010) deduziu a seguinte equação de KC generalizada:

$$
\sqrt{\frac{k}{\phi}}=\xi \frac{\phi^{(\zeta+2) / 2}}{(1-\phi)^{\eta}},
$$

onde $\zeta=D_{r}>0, \eta=D_{1 / M_{b}}, \xi=\left(C_{r}^{-1 / 2} \frac{C_{1 / M_{b}}}{f}\right) \geqslant 0$.

Essa equação foi utilizada como modelo para os ajustes entre a porosidade e permeabilidade e o algorítimo de Evolução Diferencial, descrito a seguir, como metodologia para estes ajustes.

\subsection{O algorítimo da Evolução Diferencial (ED)}

Segundo Storn e Price (1997), a ED é um método de procura direto no qual se utiliza $N_{P}$ vetores $N$-dimensionais de parâmetros, que caracteriza a população, como ilustrado na equação 6 a seguir:

$$
x_{i, G} \mid i=1,2, \cdots, N_{P} .
$$

Para esse trabalho foi utilizado $N_{P}=100$.

O algorítimo inicializa com valores aleatório ocupando todo o espaço de parâmetros. Os parâmetros utilizados nesse caso foram $\zeta, \eta$ e $\xi$, derivados da equação 5. Para meIhor entendimento desse algorítimo, a seguir será mostrado uma descrição geral, seguida de uma explicação de suas operações.

Com a população de vetores inicializada, segue-se para os passos seguintes, porém encontrada uma solução preliminar, pertubações são adicionadas a essa população. $O$ vetores mutantes que representam essa população são gerados pela diferença entre dois vetores da população, multiplicados por um fator, somado a um terceiro vetor. Posteriormente, o vetor mutante tem seus parâmetros mesclado com um vetor específico (chamado vetor alvo), gerando um vetor de teste, etapa essa chamada de recombinação. Esse vetor de teste substitui o vetor alvo se por acaso o seu conjunto de parâmetros substituídos na função custo resultar num valor menor, etapa essa chamada de seleção. Nesse trabalho a função custo foi dada pela equação 8 . Essas três etapas são repetidas $N_{P}$ vezes, para que todos os vetores da população tenham sido vetores alvo pelo menos uma vez. Desse modo, o processo como um todo é repetido iterativamente até que se chegue a um valor da função custo menor que o erro ou que um máximo de iterações predefinidas seja atingida.

As etapas de mutação, recombinação e seleção serão explicadas a seguir, assim como a proposta de modificação utilizada nesse trabalho.

\subsubsection{Mutação}

A mutação originalmente tem como entrada a população de valores, como na equação 7 , seja inicial ou resultante de uma iteração já completada, assim como o vetor que define os limites dos parâmetros e o índice do vetor alvo. Logo a seguinte operação é feita:

$$
v_{i, G+1}=x_{i_{1}, G}+F\left(x_{i_{2}, G}-x_{i_{3}, G}\right),
$$

sendo $v_{i, G+1}$ o vetor mutante e $F$ o coeficiente de mutação (STORN; PRICE, 1997).

Cada parâmetro do vetor mutante é então testado para que se mantenha dentro do espaço dos parâmetros, definido por seus limites. Assim, se o valor do parâmetro for maior que o limite superior, o valor do limite superior substitui o valor original, e o mesmo é feito de forma análoga para testar o limite inferior. No código implementado para o presente 
trabalho, foi utilizado parâmetros com limite superior igual à 15 e inferior igual a 0 .

\subsubsection{Recombinação}

A recombinação originalmente tem como entrada o vetor mutante, o vetor alvo e o coeficiente de recombinação $(C R)$. Para cada parâmetro do vetor alvo, é gerado um coeficiente de cruzamento aleatório entre 0 e 1, e caso esse valor seja menor que o $C R$, o valor do parâmetro do vetor mutante substitui o do vetor alvo. Por outro lado, se for menor, o valor do vetor alvo é mantido.

\subsubsection{Seleção}

A seleção tem como entrada a população, o vetor de teste e o índice do vetor alvo em meio a população. O primeiro passo é a avaliação feita pela função custo, equação 8 . Caso o valor dessa função, para os valores do vetor teste, seja menor que para os valores do vetor alvo, então o vetor teste é aceito na mesma posição do vetor alvo. Se o contrário acontecer ele não é aceito.

Como no algorítimo ED, a população inicial tem pouca ou nenhuma correlação, para acelerar a convergência para um mínimo global seria interessante alterar como se comportam os coeficientes de mutação e recombinação ao longo das iterações. Essa correlação é um conceito que indica o "grau de parentesco"das populações (soluções). Refletindo o quão próximos estão os indivíduos dessa população dentro do espaço dos parâmetros. Se essa correlação for baixa, considera-se que estas populações poderão estar mais longe de mínimos da função custo, logo será necessário mais recombinações para que os indivíduos "cruzem"(recombinem), com o intuito de aproximá-los desse mínimo. De forma análoga, se a correlação for alta, isso pode indicar que estes indivíduos ou soluções estejam muito próximos de mínimos locais, sendo interessante aumentar as operações de mutação para fazer com que o ED procure por outros mínimos em sua vizinhança, a fim de facilitar a localização de um mínimo global, a partir de uma melhor exploração do espaço de soluções. Assim, é interessante manter o coeficiente de recombinação alto e o de mutação baixo, no início do algorítimo, e o contrário ao final.

Para reproduzirmos a afirmação acima, no código implementado foi escolhido uma variação entre os coeficientes de mutação e recombinação de acordo com uma função linear. $O$ valores iniciais e finais para o coeficiente de mutação ficou $\left(F_{0} ; F_{f}\right)=(0,3 ; 0,5)$; e para os coeficiente de recombinação foi de $\left(C R_{0} ; C R_{f}\right)=(0,6 ; 0,3)$. Esses valores máximos e mínimos foram parametrizados em relação ao número máximo de iterações (foi utilizado $\max _{i t e r}=300$ ).

A cada geração do algorítimo, o valor mínimo da função custo foi testado. Se o mínimo for menor que o erro, então o algorítimo satisfez um dos critérios de parada e é interrompido, exportando o vetor de parâmetros que gerou o valor mínimo. $O$ erro utilizado foi $\varepsilon=10^{-3}$, de forma a evitar que se interrompesse o algorítimo prematuramente.

\subsubsection{A função custo utilizada}

A função custo utilizada é caracterizada pela seguinte equação:

$$
f(x, \phi)=\sum_{i=1}^{N P} k_{\text {calc }}\left(x, \phi_{i}\right)-k_{\text {exp }}\left(\phi_{i}\right),
$$

onde $k_{\text {calc }}$ e $k_{\text {exp }}$ são respectivamente, a permeabilidade calculada, utilizando a equação 5 , e experimental resultado de medidas laboratoriais.

\subsection{Dados utilizados}

Nessa pesquisa utilizamos dados experimentais extraídos do trabalho de Aase, Bjorkum e Nadeau (1996). Aase, Bjorkum e Nadeau (1996), analisou corpos de prova de 15 poços, utilizando métodos de microscopia de elétron (SEM), difração de raio-X (XRD), bem como análise de lâminas. Os dados contém valores de porosidade, permeabilidade, tamanho dos grãos e conteúdo de carbonato, argila, micro e macro quartzo; para uma série de profundidades. Essas medidas foram retiradas da formação de arenitos Ula e Gyda, no Mar Norte do setor Norueguês.

Esses dados foram utilizados como entrada no algorítimo ED com a equação de KC generalizada, tendo como resultado valores para os parâmetros $\zeta, \eta$ e $\xi$ em cada poço usado. Dos 15 poços, somente foram utilizadas para confecção dos gráficos resultantes dessa pesquisa, os poços de $A$ até $H$, com exceção do poço $E$, pois possui somente três pontos. Não obstante, os resultados em formato de gráfico estão exibidos na seção seguinte.

\section{Resultados}

Os resultados dessa pesquisa serão apresentados nessa seção sob forma de gráficos, que mostram os pontos experimentais, dados calculados a partir da função de permeabilidade dada pela equação 5 , utilizando os parâmetros calculados pelo algorítimo de Evolução Diferencial modificado. Antes de mostrar os resultados é importante mencionar que a equação 5 calcula a permeabilidade na unidade darcy $(d)$, e por isso os resultados da função custo $\left(f_{\text {best }}\right)$ estarão todos nesta unidade, porém para efeito de escala, os gráficos estão em milidarcy $(m d)$.

O gráfico para o poço A é mostrado na figura 1. Observa-se que a função custo é bem minimizada nesse poço, tendo $f_{\text {best }}=0,0842$. Isso é melhor notado nos valores menores de porosidade, visto que em valores de porosidade acima de aproximadamente $20 \%$ os valores calculados e experimentais começam a divergir. Além disso, a litologia desse poço tem alto conteúdo de argila e carbonato, o que pode ter contribuído para o decréscimo principalemente da permeabilidade. 


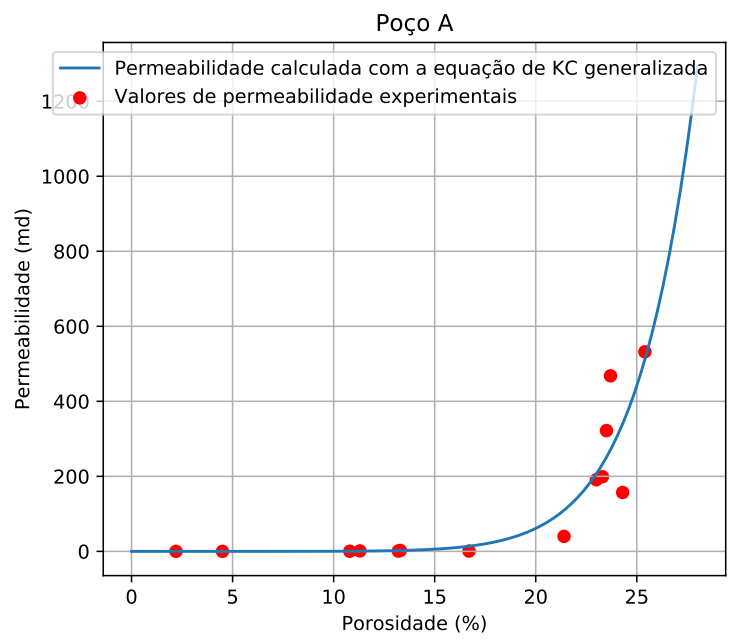

Figura 1: Gráfico com os valores de permeabilidade experimentais e calculados para o poço A, com parâmetros: $\zeta=$ $3,313454192307423, \eta=4,38340574532554$ e $\xi=15,0$.

O gráfico para o poço B é mostrado na figura 2. Da mesma forma, a função custo foi bem minimizada, com $f_{\text {best }}=$ 0,6459 , um valor cerca de 10 vezes maior que o do poço $A$. O padrão de divergência a partir de aproximadamente $20 \%$ é mantido.

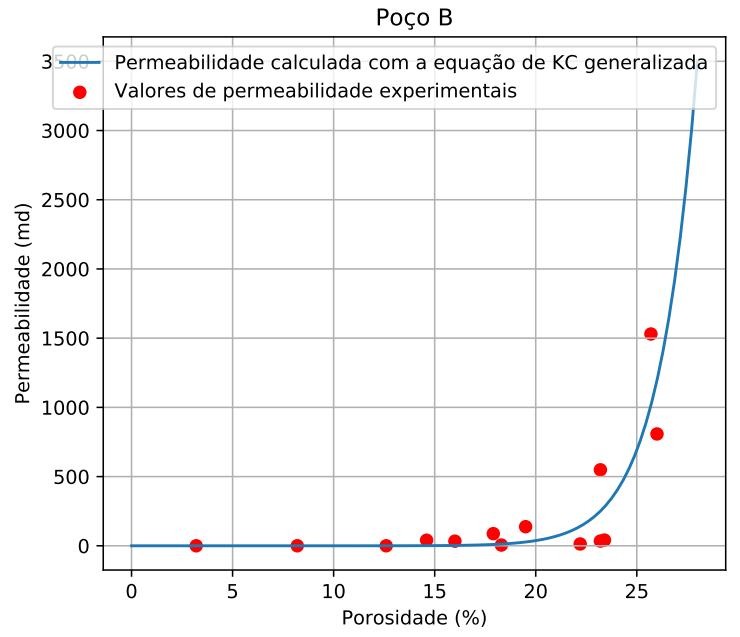

Figura 2: Gráfico com os valores de permeabilidade experimentais e calculados para o poço $\mathrm{B}$, com parâmetros: $\zeta=4,890452008391821, \eta=8,99140805982993$ e $\xi=$ 14,825945602622475 .

O gráfico para o poço C é mostrado na figura 3. A função foi bem minimizada nos dois pontos de porosidade próximos a 0,1 . Refletindo o valor de $f_{\text {best }}=0,006625$, surpreendentemente pequeno.

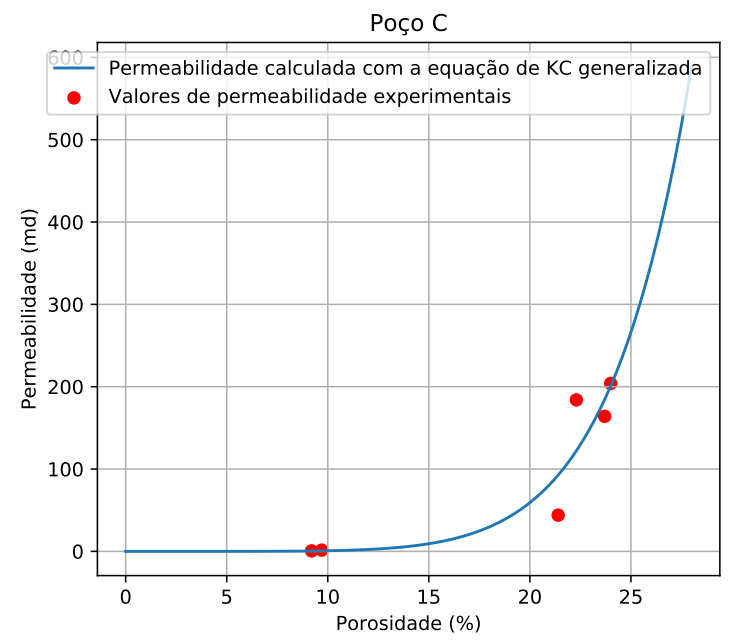

Figura 3: Gráfico com os valores de permeabilidade experimentais e calculados para o poço C, com parâmetros: $\zeta=2,628054532358561, \eta=1,9320224872268148$ e $\xi=$ 14,638369267385135 .

O gráfico para o poço D é mostrado na figura 4. Observa-se que a função foi adequadamente minimizada para valores menores que $23,5 \%$ de porosidade, no entanto, no geral $f_{\text {best }}=0,1034$. Além disso nota-se que dois dos parâmetros $\eta$ e $\xi$ convergiram para o limite superior preestabelecido.

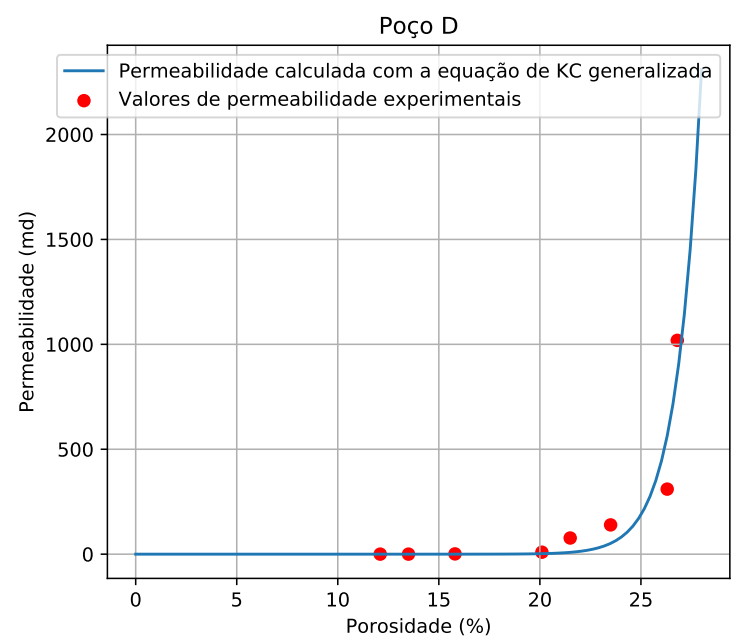

Figura 4: Gráfico com os valores de permeabilidade experimentais e calculados para o poço $\mathrm{D}$, com parâmetros: $\zeta=8,338872127921626, \eta=15,0$ e $\xi=15,0$.

O gráfico para o poço $\mathrm{F}$ é mostrado na figura 5. Apesar de ter poucos pontos com baixa porosidade $(<20 \%)$, a função foi bem minimizada, com $f_{\text {best }}=0,007566$. Além disso, foi um dos casos excepcionais em que um dos parâmetros convergiu para seu limite superior $(\xi=15)$. 


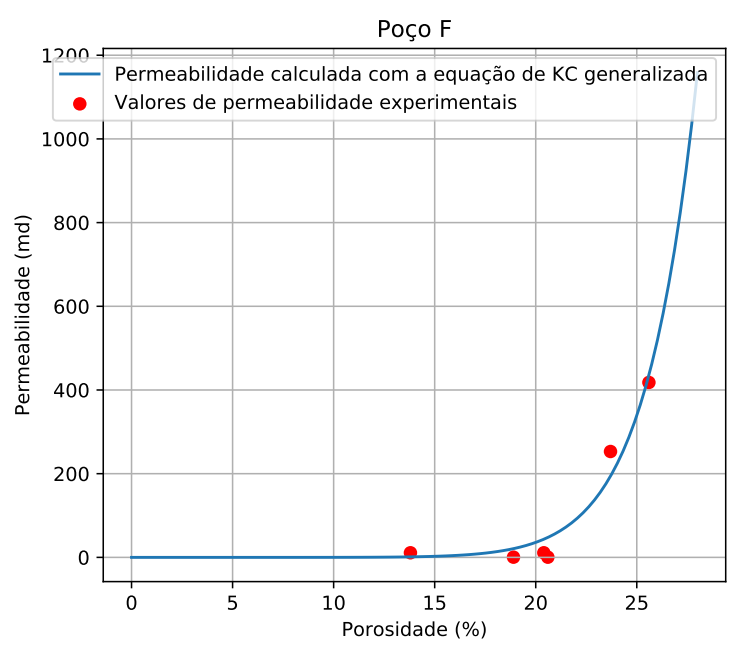

Figura 5: Gráfico com os valores de permeabilidade experimentais e calculados para o poço $\mathrm{F}$, com parâmetros: $\zeta=3,9180334351280894, \eta=5,3722852338378795 \mathrm{e}$ $\xi=15,0$.

O gráfico para o poço $\mathrm{G}$ é mostrado na figura 6 . $\mathrm{O}$ poço $\mathrm{G}$, foi um caso excepcional em que o $\eta$ zerou, e praticamente todos os pontos com porosidade abaixo de vinte estão concentrados ao redor de $\phi=14 \%$. Apesar disso, a função foi bem minimizada, com $f_{\text {best }}=0,0001838$. Esse caso é parecido com o do poço $\mathrm{C}$ na figura 3 .

Poço G

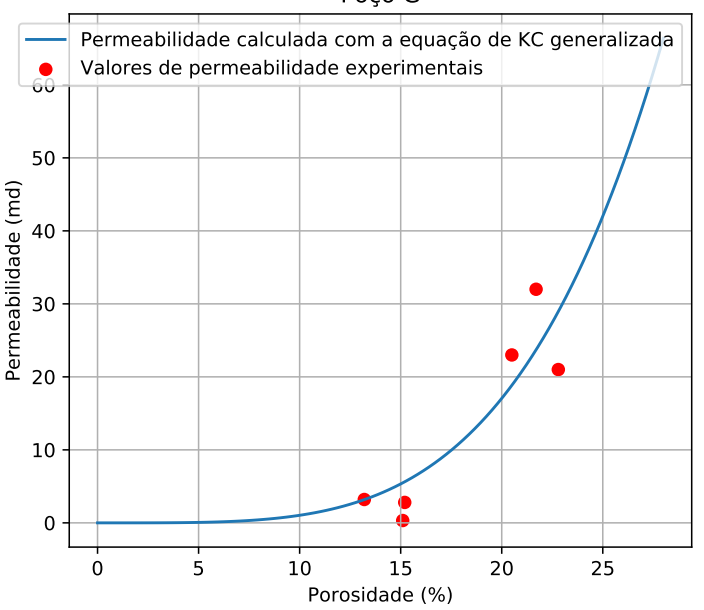

Figura 6: Gráfico com os valores de permeabilidade experimentais e calculados para o poço $\mathrm{G}$, com parâmetros: $\zeta=$ $1,0645291801614014, \eta=0,0$ e $\xi=3,4401998671109384$.

O gráfico para o poço H é mostrado na figura 7. Esse poço é peculiar, pois apesar de todos os pontos serem próximos à $20 \%$, a função foi bem minimizada, com $f_{\text {best }}=0,07964$. É importante salientar que, junto ao poço $\mathrm{A}$, o poço $\mathrm{H}$ é o que tem uma litologia com maior conteúdo de carbonatos somado à argila, quando comparados com os outros poços analisados nesse trabalho.

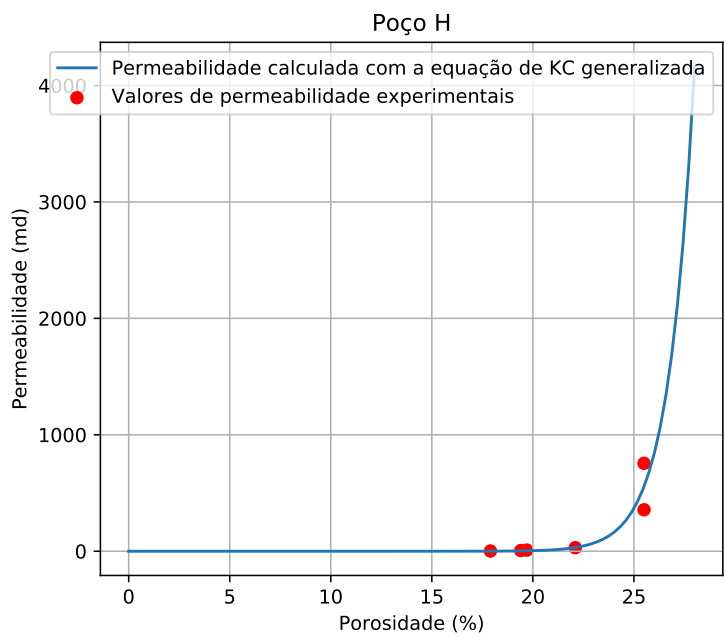

Figura 7: Gráfico com os valores de permeabilidade experimentais e calculados para o poço $\mathrm{H}$, com parâmetros: $\zeta=7,291594307586502, \eta=14,871951729297168$ e $\xi=$ 10,584394703483005 .

\section{Discussão e Conclusões}

Em suma as amostras de rochas adquiridas a partir dos poços analisados, eram formadas por arenitos com alto teor de carbonatos e argila, como pode ser visto no trabalho de Aase, Bjorkum e Nadeau (1996). Podendo contar com a cimentação de quartzo em alguns trechos. Assim, nos trechos em que há maior teor de argila, carbonatos ou cimentação, a tendência é que a permeabilidade fique mais próxima de zero. De acordo com a distribuição da argila/foIhelho contida nas amostras analisadas, de origem detrítica (laminar, disperso ou estrutural) ou autigênica, ela poderá contribuir também para a condutividade do reservatório ao qual estas amostras pertencem. Logo, o meio poroso dessas rochas é mais complexo que o do arenito limpo. Por essa razão, o uso de um modelo que melhor caracterize essa complexidade foi necessário.

A equação de Kozeny-Carman generalizada é mais eficiente para esses casos, justamente por assumir leis fractais que abrangem um nível de complexidade maior. Por isso a minimização utilizando-a como base, foi efetiva na maior parte dos poços analisados. Isso pode ser especialmente notado nos poços $\mathrm{A}$ e $\mathrm{H}$, nas figuras 1 e 2 , onde mesmo em litologias com alto teor de argila e carbonatos, os pontos foram bem ajustados. Além disso, os valores dos três parâmetros que o modelo ajustado apresentou $(\zeta, \eta \mathrm{e}$ $\eta)$, possuem siginficado físico e se relacionam diretamente com a tortuosidade e a superfície específica do material poroso, as quais dependem entre outros fatores, de modo complexo, da forma e da dimensão dos grãos, de sua classificação granulométrica, dos graus de compactação e cimentação, sendo assim, o entendimento desse relacionamento poderá ajudar na simulação do campo de permoporosidade desses reservatórios. 


\section{Agradecimentos}

Os autores deste trabalho agradecem ao suporte técnico ao Centro de pesquisa em Geolgia e Geofísica da Universidade Federal da Bahia pelo suporte técnico.

\section{Referências}

AASE, Nils Einar; BJORKUM, Per Arne; NADEAU, Paul H. The effect of grain-coating microquartz on preservation of reservoir porosity. AAPG bulletin, American Association of Petroleum Geologists, v. 80, n. 10, p. 1654-1673, 1996.

BRÊTTAS, Juan Diego Cardoso. Geração de meios porosos fractais com uma nova equação do tipo Kozeny-Carman. 2010. Dissertação de Mestrado Universidade do Estado do Rio de Janeiro, Nova Friburgo, 2010.

BUST, Vivian $\mathrm{K}$ et al. The petrophysics of shale gas reservoirs: Technical challenges and pragmatic solutions. Petroleum Geoscience, Geological Society of London, v. 19, n. 2, p. 91-103, 2013.

CARMAN, Philip Crosbie. Determination of the specific surface of powders I. Transactions. J. Soc. Chemical Industries., v. 57, p. 225-234, 1938.

. Fluid flow through granular beds. Trans. Inst. Chem. Eng., v. 15, p. 150-166, 1937.

CHAPUIS, Robert P; AUBERTIN, Michel. Predicting the coefficient of permeability of soils using the Kozeny-Carman equation. École polytechnique de Montréal, 2003.

COSTA, Antonio. Permeability-porosity relationship: A reexamination of the Kozeny-Carman equation based on a fractal pore-space geometry assumption. Geophysical

Research Letters, v. 33, 2 2006. DOI:

10.1029/2005gl025134.

KOZENY, Josef. Uber kapillare leitung der wasser in boden. Royal Academy of Science, Vienna, Proc. Class I, v. 136, p. 271-306, 1927.

SEELHEIM, F. Methoden zur Bestimmung der Durchlässigkeit des Bodens. Analytical and Bioanalytical Chemistry, Springer, v. 19, 1 1880. DOI: 10.1007/bf01341054.

STORN, Rainer; PRICE, Kenneth. Differential Evolution A Simple and Efficient Heuristic for global Optimization over Continuous Spaces. Journal of Global Optimization, Springer US, v. 11, 4 dez. 1997. DOI: 10.1023/a:1008202821328.

YU, Gang et al. Rock physics diagnostics and modeling for shale gas formation characterization in China. In: UNCONVENTIONAL RESOURCES TECHNOLOGY CONFERENCE (URTEC), 2014, USA. 